\title{
Reactivation of Hepatitis B Virus in Rheumatologic Patients Receiving Immunosuppressive Agents
}

Feng-Chiao Tsai • Song-Chou Hsieh •

Ding-Shinn Chen · Jin-Chuan Sheu • Chien-Hung Chen

Published online: 23 January 2007

(C) Springer Science+Business Media, LLC 2007

The author name "Ding-Shinn Chen" has been repeated in both the list of author names below the article title and the list of author names in the affiliation footnote. The author

name "Chien-Hung Chen" should replace the second "DingShinn Chen" in both lists of author names. Thus, C.-H. Chen (not D.-S. Chen) is the corresponding author.

The online version of the original article can be found at http://dx.doi.org/10.1007/s10620-006-9074-8

F.-C. Tsai · S.-C. Hsieh · D.-S. Chen · J.-C. Sheu · C.-H. Chen ( $₫)$ Department of Internal Medicine, National Taiwan University Hospital and National Taiwan University College of Medicine, 7 Chung-Shan South Road, Taipei 100, Taiwan

e-mail: chenhcc@ha.mc.ntu.edu.tw 